\title{
The plasma exosomal miR-1180-3p serves as a novel potential diagnostic marker for cutaneous melanoma
}

\author{
Yeye Guo ${ }^{1,2,3+}{ }^{+}$Xu Zhang ${ }^{1,2,3+}$, Linconghua Wang ${ }^{4}$, Min Li ${ }^{4}$, Minxue Shen ${ }^{1,2,3}$, Zhe Zhou ${ }^{1,2,3}$, Susi Zhu ${ }^{1,2,3}$, \\ Keke Li $\mathrm{Li}^{1,2,3}$, Zhiqin Fang ${ }^{1,2,3}$, Bei Yan ${ }^{1,2,3}$, Shuang Zhao ${ }^{1,2,3^{*}+}$, Juan Su $\mathrm{Su}^{1,2,3^{*+}}$, Xiang Chen ${ }^{1,2,3^{*+}}$ and \\ Cong Peng ${ }^{1,2,3^{*}+}$ (D)
}

\begin{abstract}
Background: Exosomes are a promising tool in disease detection because they are noninvasive, cost-effective, sensitive and stable in body fluids. MicroRNAs (miRNAs) are the main exosomal component and participate in tumor development. However, the exosomal miRNA profile among Asian melanoma patients remains unclear.

Methods: Exosomal miRNAs from the plasma of melanoma patients $(n=20)$ and healthy individuals $(n=20)$ were isolated and subjected to small RNA sequencing. Real-time PCR was performed to identify the differential miRNAs and to determine the diagnostic efficiency. Proliferation, scratch and Transwell assays were performed to detect the biological behavior of melanoma cells.

Results: Exosomal miRNA profiling revealed decreased miR-1180-3p expression as a potential diagnostic marker of melanoma. The validation group of melanoma patients $(n=28)$ and controls $(n=28)$ confirmed the diagnostic efficiency of miR-1180-3p. The level of miR-1180-3p in melanoma cells was lower than that in melanocytes. Accordingly, the level of miR-1180-3p was negatively associated with the proliferation, migration and invasion of melanoma cells. Functional analysis and target gene prediction found that ST3GAL4 was a potential target and highly expressed in melanoma tissues and was negatively regulated by miR-1180-3p. Knockdown of ST3GAL4 hindered the malignant phenotype of melanoma cells.
\end{abstract}

Conclusions: This study indicates that reduced exosomal miR-1180-3p in melanoma patient plasma is a promising diagnostic marker and provides novel insight into melanoma development.

Keywords: Melanoma, Plasma, Exosome, miR-1180-3p, Diagnostic marker, ST3GAL4

\section{Background}

For years, researchers have been devoted to developing

\footnotetext{
*Correspondence: shuangxy@csu.edu.cn; sujuanderm@csu.edu.cn; chenxiangck@126.com; pengcongxy@csu.edu.cn

'Yeye Guo and Xu Zhang contributed equally to this work

†Shuang Zhao, Juan Su, Xiang Chen and Cong Peng contributed equally to this work

1 Department of Dermatology, Xiangya Hospital, Central South University, Xiangya Road \#87, Changsha 410008, China
}

Full list of author information is available at the end of the article liquid biopsy, an ideal approach to molecular cancer diagnosis at an early stage due to its high specificity and noninvasiveness for cancer diagnosis [1]. Circulating tumor cells, DNAs and exosomes are the main targets for liquid biopsy [2]. Among them, exosomes have exceptional advantages in several aspects. Exosomes are $30-150 \mathrm{~nm}$ extracellular vesicles secreted by almost all cells and can be detected in various biological fluids [3]. Owing to their tiny size and biocompatibility, exosomes can remain stable in the circulation for a long time [4]. These characteristics 
make exosomes particularly promising in liquid biopsies [5]. Proteins, metabolites, and nucleic acids are contained in exosomes [6]. MicroRNAs (miRNAs) are noncoding RNAs that are critical exosomal constituents. MiRNAs are processed from precursor molecules (pri-miRNAs) by RNA polymerases II and III, followed by a series of cleavage events [7]. The disorder of miRNAs is associated with various diseases, including cancers, as they regulate the post-transcription of target genes [8] and affect metabolic and cellular pathways, including those related to cell proliferation, differentiation and survival.

Exosomal miRNAs play critical roles in the diagnosis and prognosis of various cancers $[9,10]$, including melanoma. Cutaneous melanoma is the most severe skin cancer due to its aggressiveness and tendency to metastasize [11]. The five-year survival rate of melanoma varies from over $90 \%$ in the localized stage to less than $20 \%$ in the advanced stage [12]. When distant metastasis occurs, the median survival is only 6 to 9 months, with a 5 -year survival rate of less than $5 \%$ [13]. The considerable variation in survival indicates the urgency to screen melanoma patients in the early stages. A miRNA array was generated in plasma-derived exosomes from familial melanoma patients (CDKN2A/p16 gene carriers), nonfamilial melanoma patients and healthy control subjects. Numerous miRNAs have been identified, including miR-17, miR19a, and miR-21, in metastatic melanoma [14]. The level of exosomal miR-125b in serum was also demonstrated to be relatively high in patients with advanced melanoma. However, most previous studies were performed on Caucasian patients, and exosomal miRNA alterations in Asian melanoma patients remain unknown. The genetic changes, clinical features, anatomical origin and prognosis of melanoma patients vary among different populations [15]. Therefore, identifying differential exosomal miRNAs in the Asian group is highly desirable. In the current study, we described the plasma exosomal miRNA signatures among Chinese melanoma patients to identify a potential diagnostic target and the downstream pathway for this group of patients.

\section{Methods}

\section{Patient plasma samples}

Healthy donors $(n=48)$ and melanoma patients $(n=48)$ were recruited at Xiangya Hospital, Central South University, following the Declaration of Helsinki. All the donors have signed the consent forms. This study was approved by the Institutional Research Ethics Board of Xiangya Hospital, Central South University. Five milliliters $(\mathrm{mL})$ of whole blood was collected from each subject into ethylenediaminetetraacetic acid (EDTA) tubes and then centrifuged at $3000 \times g$ for $20 \mathrm{~min}$ at $4{ }^{\circ} \mathrm{C}$. After centrifugation, plasma samples were collected and immediately stored at $-80^{\circ} \mathrm{C}$ until use. The clinical characteristics are shown in Additional file 4: Table S1.

\section{Exosome isolation}

Plasma $(1.5 \mathrm{~mL})$ was centrifuged at $16,000 \times g$ for $30 \mathrm{~min}$ at $4{ }^{\circ} \mathrm{C}$ to remove the microvesicles. The supernatant was subjected to exoRNeasy kits (77144, QIAGEN, Hilden, Germany) according to the manufacturer's protocol to isolate RNAs from exosomes. Briefly, samples were mixed with Buffer XBP and subjected to an exoEasy spin column. The device was centrifuged for $1 \mathrm{~min}$ at $500 \times g$, and the column was washed with Buffer XWP. Then, $700 \mu \mathrm{L}$ of QIAzol was added to the membrane and centrifuged for $5 \mathrm{~min}$ at $5000 \times g$ to collect the lysate. Ninety microliters of chloroform was added to the lysate, shaken, and then incubated for $2 \mathrm{~min}$ at room temperature. The sample was centrifuged at $12,000 \times g$ for $15 \mathrm{~min}$ at $4{ }^{\circ} \mathrm{C}$. The upper aqueous phase was collected and mixed with 2 volumes of $100 \%$ ethanol and then subjected to an $\mathrm{RNe}$ asy MinElute spin column. The sample was centrifuged at $\geq 8000 \times g(\geq 10,000 \mathrm{rpm})$ for $15 \mathrm{~s}$ at room temperature. The spin column was washed with $700 \mu \mathrm{L}$ of Buffer RWT and $500 \mu \mathrm{L}$ of Buffer RPEto on an RNeasy MinElute spin column sequentially. Then, $14 \mu \mathrm{L}$ of RNase-free water was added to the spin column membrane to elute the RNA.

\section{Cell lines and cell culture}

The human melanoma cell lines ME4405, A375, SKMel-5 and Sk-Mel-28 (maintained in our laboratory) and the human melanocyte cell line PIG1 (a gift from the Department of Dermatology, Xiangya Third Hospital) were used in this study. Melanoma cells were grown in DMEM (01-052-1A, Biological Industries, Beit HaEmek, Israel) or Opti-MEM for PIG1 (31985070, Gibco, Waltham, MA) supplemented with $10 \%$ fetal bovine serum (04-001-1ACS, Biological Industries) and 1\% penicillin-streptomycin (03-031-1B, Biological Industries) at $37^{\circ} \mathrm{C}$ and $5 \% \mathrm{CO}_{2}$.

\section{Cell proliferation assays}

Cell viability was assessed using a Cell Counting Kit-8 (CCK-8) assay (Bimake, Houston, TX) according to the manufacturer's instructions. Cells were seeded into 96-well plates at 3000 cells/well and cultured for 24, 48, 72 , or $96 \mathrm{~h}$. Then, $10 \mu \mathrm{L}$ of CCK-8 solution was added to each well, and the 96-well plate was incubated for $2 \mathrm{~h}$ at $37{ }^{\circ} \mathrm{C}$ and $5 \% \mathrm{CO}_{2}$. The fluorescence of each plate was measured using a spectrophotometer at an emission wavelength of $450 \mathrm{~nm}$ (Beckman, Brea, CA). Six replicates per sample were analyzed. 


\section{Cell transfection}

Cells were transfected with a miR-1180-3p mimic or inhibitor (GeneCopoeia, Rockville, MD) using TurboFect Transfection Reagent (R0533, Thermo Scientific, and incubated for $20 \mathrm{~min}$. The mixture was added to growing cells for 36 to $48 \mathrm{~h}$ to facilitate transfection.

\section{Plasmid and lentiviral vector construction}

Lentivirus plasmids containing pLKO.1, pSPAX2 and pMD2G and ST3GAL4 shRNAs were purchased from Thermo Scientific (MA, USA) and GeneChem (Shanghai, China). To establish stable ST3GAL4-knockdown cells, pLKO.1-shST3GAL4 or pLKO.1-shMock plasmids were cotransfected with packaging plasmids (pSPAX2 and PMD2G) into 293T cells. The supernatant fractions containing lentiviral particles were collected separately at 48 and $72 \mathrm{~h}$, and melanoma cells were infected with lentiviral particles in medium supplemented with $10 \mu \mathrm{g} /$ $\mathrm{mL}$ polybrene. At $16 \mathrm{~h}$ after infection, the medium was replaced with fresh medium containing a suitable concentration of puromycin. The appropriate experiments were performed with these cells until all control cells (uninfected) were dead (usually 36-48 h) in puromycincontaining medium.

\section{Transwell assay}

For the invasion assay, a Transwell experiment was performed with 8- $\mu \mathrm{m}$-pore size chambers inserted into 24-well plates (Corning, NY, USA). Matrigel (BD Biosciences, NJ) was diluted (1:7) in serum-free DMEM and then added to each chamber and allowed to solidify completely. Transwell migration assays were performed without Matrigel. Transfected cells were obtained, resuspended in serum-free medium at a concentration of $4 \times 10^{4} / 100 \mu \mathrm{L}$ and seeded in the upper chambers, while $550 \mu \mathrm{L}$ of DMEM containing 30\% FBS was placed into the bottom chamber as a chemotactic factor. After 24 or $48 \mathrm{~h}$, the cells were fixed with $4 \%$ paraformaldehyde for $15 \mathrm{~min}$ at room temperature. Nonmotile or noninvaded cells on the top surface of the filter were removed, while motile or invaded cells on the bottom surface were stained with crystal violet. ImageJ software was used to quantify the invaded and migrated cells. The cells in three randomly selected fields (left, middle, right) per well were counted with an inverted microscope system (Ti-S, Nikon, Tokyo, Japan).

\section{Scratch assay}

Cells in complete medium were seeded in a 6-well plate at a density of $1 \times 10^{5}$ cells/well, and a straight line was scratched on the cell monolayer with a $200 \mu \mathrm{L}$ pipette tip. Then, the cells were washed with PBS three times to remove debris. Finally, the cells were cultured in DMEM supplemented with $10 \% \mathrm{FBS}$ and imaged at $24 \mathrm{~h}$ and $48 \mathrm{~h}$.

\section{Small RNA sequencing}

Small RNA library construction, library purification and sequencing were implemented according to the Wuhan Huada Sequencing Company's instructions (www.genom ics.org.cn, BGI, Shenzhen, China).

\section{Quantitative real-time PCR analysis}

Total RNA was extracted from the cells with TRIzol reagent. Exosomal RNAs were extracted by exoRNeasy kits as described above. One microgram (cells) or $10 \mathrm{ng}$ (exosomes) of total RNA was used as the template for the reverse transcription reaction (miRNA First-Strand cDNA Synthesis Kit for miRNA qPCR array, QP018, GeneCopoeia; SuperScript III First-Strand Synthesis System for Reverse Transcription PCR, 18080051, Invitrogen, Waltham, MA). The PCR primers for miR-1180-3p and U6 were purchased from GeneCopoeia, and the other primers used in this study were as follows:

\section{ST3GAL4: Forward 5'GTCGTATTGGAGACCGTC $A A G^{\prime}$ \\ Reverse $\quad 5^{\prime} C T T G G A A G G G A G A A A A G G$ TGAG'. \\ GAPDH: Forward 5'GTATCGTGGAAGGACTCA TGAC. \\ Reverse $5^{\prime} A C C A C C T T C T T G A T G T C A$ TCAT.}

Real-time PCR was performed using a miRNA qRTPCR Detection Kit (QP016, GeneCopoeia) or 2X SYBR Green qPCR Master Mix (B21702, Bimake, Houston, TX). qRT-PCR assays and data collection were performed on a QuantStudio 3 real-time PCR system (Applied Biosystems, Waltham, MA). Data were analyzed

\footnotetext{
(See figure on next page.)

Fig. 1 Characterization of circulating exosomes in melanoma. A Distribution of mappable small RNAs by small RNA sequencing in exosomes from plasma of melanoma patients ( $M M, n=20)$ and healthy donors $(N O, n=20)$. Small RNA sequencing was performed as described in Methods. B Volcano plot of the differentially expressed exosomal miRNAs between two groups. Significantly downregulated genes are in blue (sig=True), and significantly up-regulated genes are in red (sig=True). Non-significant genes are in black (sig=False). Black vertical lines highlight log fold changes of -1 and 1 , while Black horizontal line represents a padj of 0.001
} 


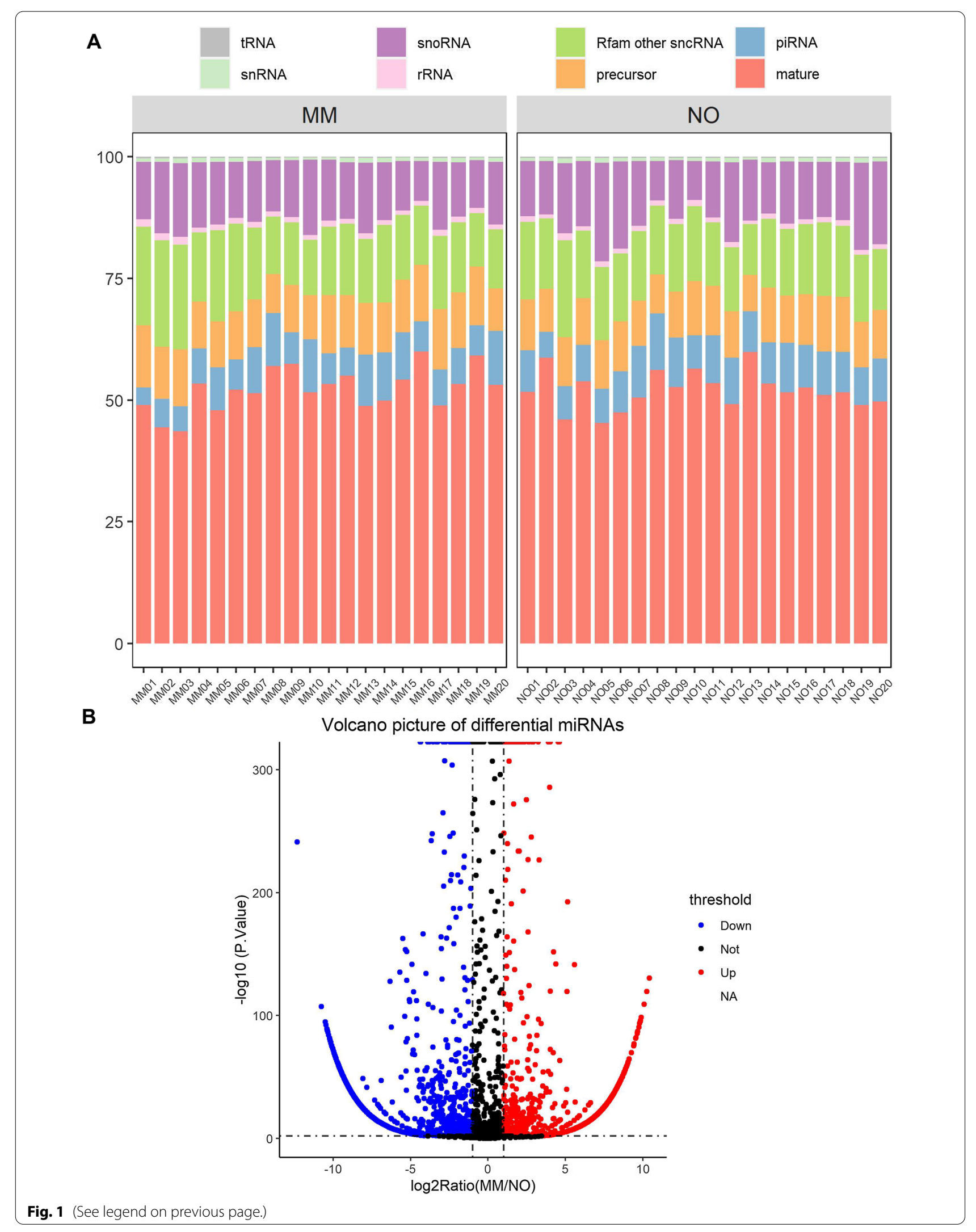




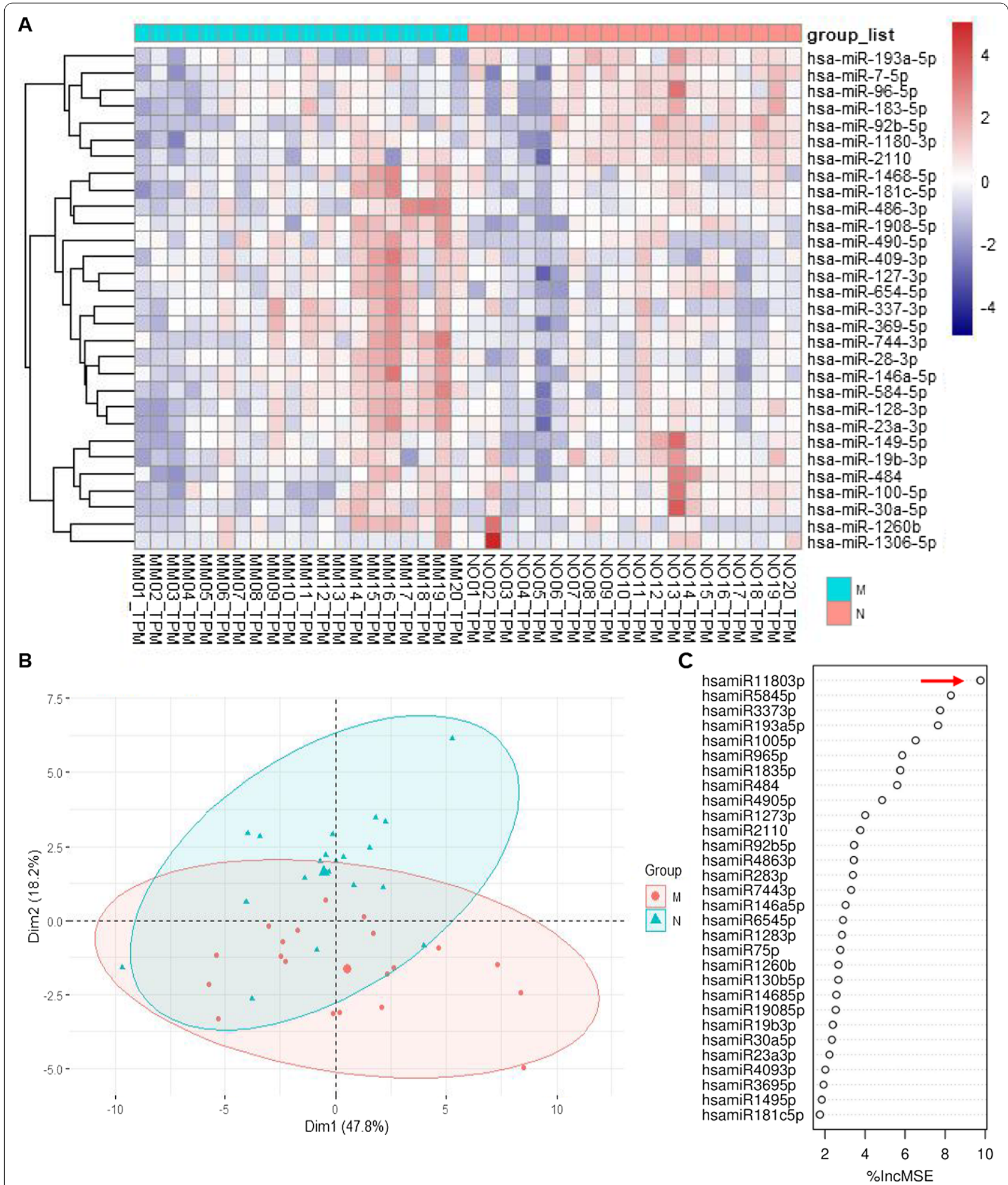

Fig. 2 Random Forest analysis of differential miRNAs. A Heat map of small RNA sequencing analysis for 30 selected genes by Random Forest algorithm. The heat map shows log 10 FPKM values for 30 selected genes (rows) and 40 samples (columns). miRNAs and samples were hierarchically clustered based on Euclidean distance of z-score data and average linkage (dendrogram not shown for samples). B Principal component analysis (PCA) of exosomal miRNA expression. PCA for miRNA expression in two groups (30 differentially expressed miRNAs; FDR-adjusted $p \leq 0.05$ ). C MiRNAs selection via Random Forest analyses ordered by the importance of contribution towards melanoma development 

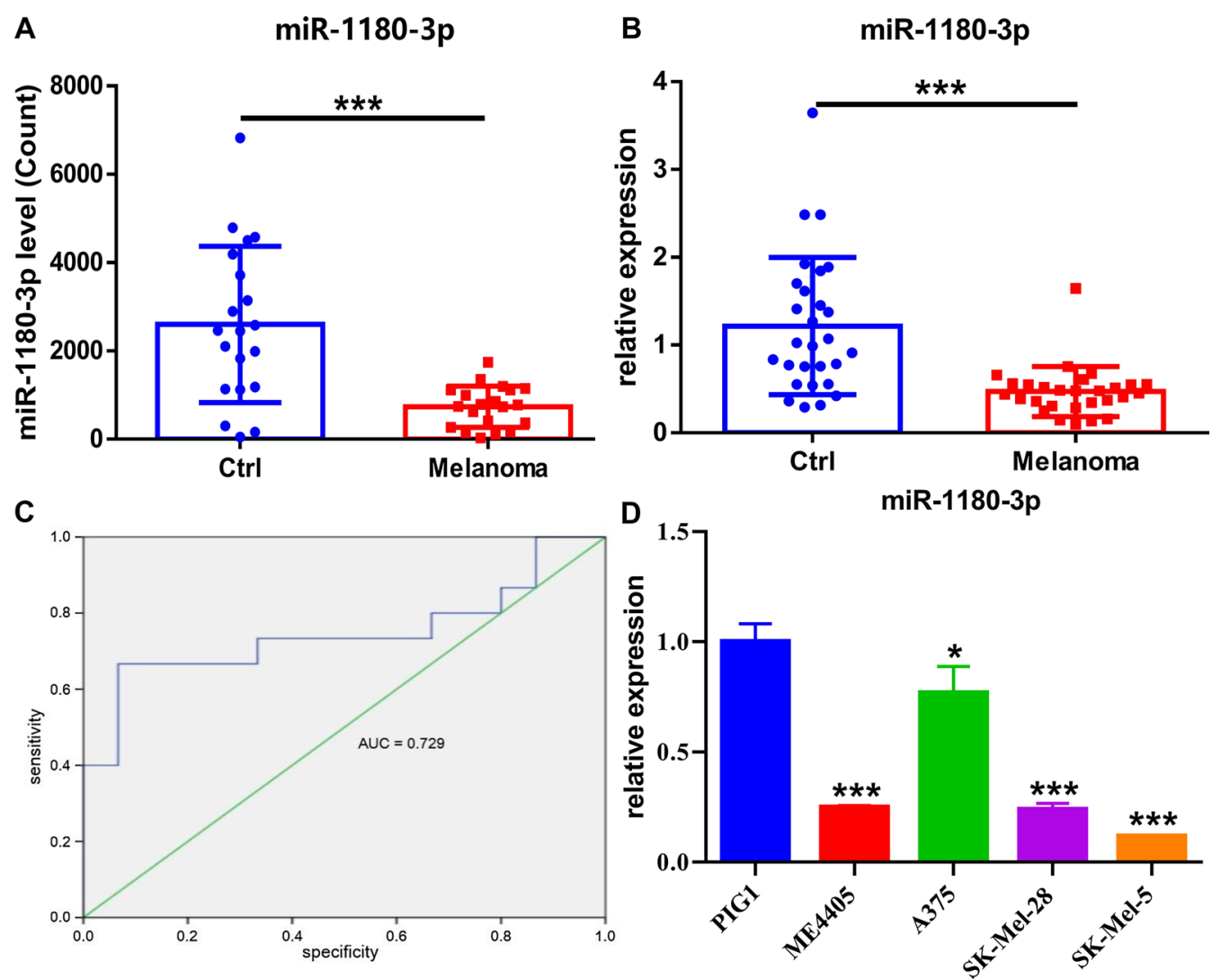

Fig. 3 miR-1180-3p is reduced in exosomes from plasma of melanoma patients and in melanoma cells. A Exosomal miR-1180-3p is downregulated in melanoma patients. The reads count of miR-1180-3p in small RNA sequencing was detected as described in Methods. Significant difference was evaluated using Student's t-test, ${ }^{*} p<0.05,{ }^{* *} p<0.01,{ }^{* * *} p<0.001$. B Exosomal miR-1180-3p is decreased in the validation cohort of melanoma patients. Exosomal RNA was extracted from plasma of melanoma patients $(n=28)$ and healthy individuals $(n=28)$, and RT-Q-PCR was performed as described in Methods. Data from multiple experiments are expressed as the mean $\pm \operatorname{SD}(n=3)$. Significant differences were evaluated using Student's t-test, ${ }^{*} p<0.05,{ }^{* *} p<0.01,{ }^{* * *} p<0.001$. C ROC curve of miR-1180-3p. The curve was constructed by the expression value for miR-1180-3p. The Area Under the Curve (AUC) with 95\% CI was computed and shown for ROC curve. The Wilcoxon-Mann-Whitney test was used to test the null hypothesis that the AUC is equal to .5 (i.e., no predictive power) and the P values for each test were shown. $\mathbf{D}$ miR-1180-3p is decreased in melanoma cells. Total RNAs were extracted from melanocyte (PIG1) and melanoma cells (ME4405, A375, SK-Mel-28, SK-Mel-5), and RT-Q-PCR was performed as described in Methods. Data from multiple experiments are expressed as the mean $\pm S D(n=3)$. Significant differences were evaluated using one-way ANOVA, ${ }^{*} p<0.05,{ }^{* *} p<0.01,{ }^{* * *} p<0.001$

using the $2^{-\triangle \triangle C T}$ values. U6 or GAPDH was used as the control.

\section{Luciferase reporter gene assays}

HEK293T cells were transfected with the pGL3ST3GAL4-WT or pGL3-ST3GAL4-Del luciferase reporter plasmid (constructed in our lab) and pRLTK, the Renilla luciferase control reporter vector (P100001, Promega, USA), and then treated with the miR-1180-3p mimic or control. After $24 \mathrm{~h}$ of transfection, the firefly and Renilla luciferase activities in the cell lysates were analyzed with a dual luciferase assay kit (Promega, Madison, WI) according to the manufacturer's protocol. For each transfection, the luciferase activities of four replicates were averaged.

\section{Bioinformatics analysis}

The following reads were filtered from the raw data of each sample: (1) low-quality reads, (2) reads with $5^{\prime}$ primer contaminants or without $3^{\prime}$ primers, (3) reads without the insert, (4) reads with poly A bases, and (5) reads shorter than $18 \mathrm{nt}$. After filtering, the remaining clean data were stored as FASTQ Files. Clean sequencing reads were aligned to the reference genome (hg38) and then annotated by AASRA [16]. The aligned data were mapped to known miRNAs 
using miRBase version 21. Differential miRNA expression was assessed using DEGseq [17]. Then, random forest was applied to the DEGseq results to identify significantly differentially expressed miRNAs. The target genes of miRNAs were predicted through miRWalk 2.0 [18], ComiR [19], and miR_ Pathway [20]. The expression profiles and prognostic significances of target genes were analyzed by GEPIA [21] and the Human Protein Atlas (https://www.prote inatlas.org/).

\section{Statistical analysis methods}

Statistical results are presented as the means \pm SDs, and significant differences were analyzed by Student's t-test or one-way ANOVA. P values $<0.05$ were considered statistically significant. Receiver operating characteristic (ROC) curves were constructed using the expression value of miR-1180-3p. The area under the curve (AUC) with the 95\% CI was calculated. The Wilcoxon-Mann-Whitney test was used to test the null hypothesis that the AUC was equal to 0.5 .

\section{Results}

\section{Characterization of circulating exosomes in melanoma}

\section{patients}

Exosomal miRNAs from the plasma of melanoma patients $(\mathrm{n}=20)$ and healthy individuals $(\mathrm{n}=20)$ were isolated and then subjected to miRNA sequencing. Over $50 \%$ of the mappable RNAs were miRNAs $(52.17 \%$ in melanoma and $51.99 \%$ in healthy donors). The rest of the RNAs were represented as transfer RNAs (tRNAs), small nuclear and nucleolar RNAs (snRNAs and snoRNAs), ribosomal RNAs (rRNAs), Rfam other small noncoding RNAs (sncRNAs), and precursor and Piwi-interacting RNAs (piRNAs), as shown in Fig. 1A. The distributions of exosomal small RNAs in the plasma of melanoma patients and controls were not significantly different. Notably, miRNAs account for a substantial portion of exosomes. Therefore, we focused on the differential miRNAs between melanoma patients and healthy donors that might be predictive of prognosis.

In total, 9703 differential miRNAs, including 666 identified miRNAs and 9037 novel miRNAs, were found by sequencing. The volcano plot describes the 666 identified miRNAs with an adjusted $p$ value $<0.05$ (Fig. 1B). The $y$-axis represents the negative log 10 value ( $P$ value), and the $\mathrm{x}$-axis presents the $\log 2$ ratio of melanoma patients vs. healthy donors $(\mathrm{MM} / \mathrm{NO})$. The upregulated miRNAs are shown as red dots $(\log 2 \mathrm{FC}>1)$, while the downregulated miRNAs are shown as blue dots $(\log 2$ ratio $(\mathrm{MM} /$ $\mathrm{NO})<-1$ ).

To better identify the miRNAs, we used random forest (RF) plots to analyze the 666 known miRNAs, revealing 30 significantly altered miRNAs. Using these 30 differential miRNAs, clustering and principal component analysis (PCA) were generated to allocate samples into groups with similar exosomal miRNA expression patterns (Fig. 2A and B). Notably, miR-1180-3p exhibited the highest score in random forest analysis, indicating that it may be vitally important for melanoma development (Fig. 2C).

\section{miR-1 180-3p expression is reduced in exosomes derived from the plasma of melanoma patients and in melanoma cells}

Therefore, we examined the sequencing data of miR$1180-3 p$, which was decreased by nearly fourfold in melanoma patients compared with the controls (Fig. 3A). To further verify the alteration, we detected the expression of plasma exosomal miR-1180-3p in another validation cohort of participants $(n=56)$. This result was consistent with the sequencing data, showing that exosomal miR1180-3p expression was decreased in the plasma of melanoma patients (Fig. 3B). The expression level of exosomal miR-1180-3p in plasma was then detected to examine its diagnostic power for melanoma. Notably, exosomal miR1180-3p exhibited an area under the ROC curve (AUC) value of 0.729 , suggesting its promise as a predictive model (Fig. 3C).

miR-1180-3p is dysregulated in hepatocellular carcinoma [22] and lung adenocarcinoma [23], while its role

\footnotetext{
(See figure on next page.)

Fig. 4 miR-1180-3p negatively regulates melanoma cells' growth, migration and invasion. A Interfere with the expression of miR-1180-3p in melanoma cells. RNAs were extracted from SK-Mel-28 infected with mimic and inhibitor of miR-1180-3p, and RT-Q-PCR was performed as described in Methods. Data from multiple experiments are expressed as the mean \pm SD $(n=3)$. Significant differences were evaluated using Student's t-test, ${ }^{*} p<0.05,{ }^{* *} p<0.01,{ }^{* *} p<0.001$. B miR-1180-3p negatively regulates the growth of melanoma cells. SK-Mel-28 transfected with mimic or inhibitor of miR-1180-3p were seeded into 96-well plates, and cell viability was examined by CCK-8 kit as described in Methods. Data from multiple experiments are expressed as the means $\pm S D$. Significant differences were evaluated using Two-way ANOVA, ${ }^{*} p<0.05,{ }^{* *} p<0.01,{ }^{* * *} p<0.001$. C, D miR-1180-3p regulates melanoma cell migration. The scratch assay was performed as described in Methods. Representative images were taken at indicated hours ( $\mathbf{C}$ and $\mathbf{D}$ upper panel) and bar chart graphs shown are from three independent experiments (C and $\mathbf{D}$ lower panel). Data are presented as the mean $\pm S D(n=3)$. Significant differences were evaluated using one-way ANOVA, ${ }^{*} p<0.05,{ }^{* *} p<0.01,{ }^{* * *} p<0.001$. E-H miR-1 180-3p regulates the invasion ability of melanoma cells. Transwell assays were performed as described in Methods. Representative images were taken at indicated hours ( $\mathbf{E}$ and $\mathbf{G}$ ). The number of invasive cells per field was calculated, and the data was presented as the means \pm SD $(n=4)$ of each group $(\mathbf{F}$ and $\mathbf{H})$. The significant difference between cells was evaluated by Student's t-test. ${ }^{*} p<0.05,{ }^{* *} p<0.01,{ }^{* * *} p<0.001$
} 

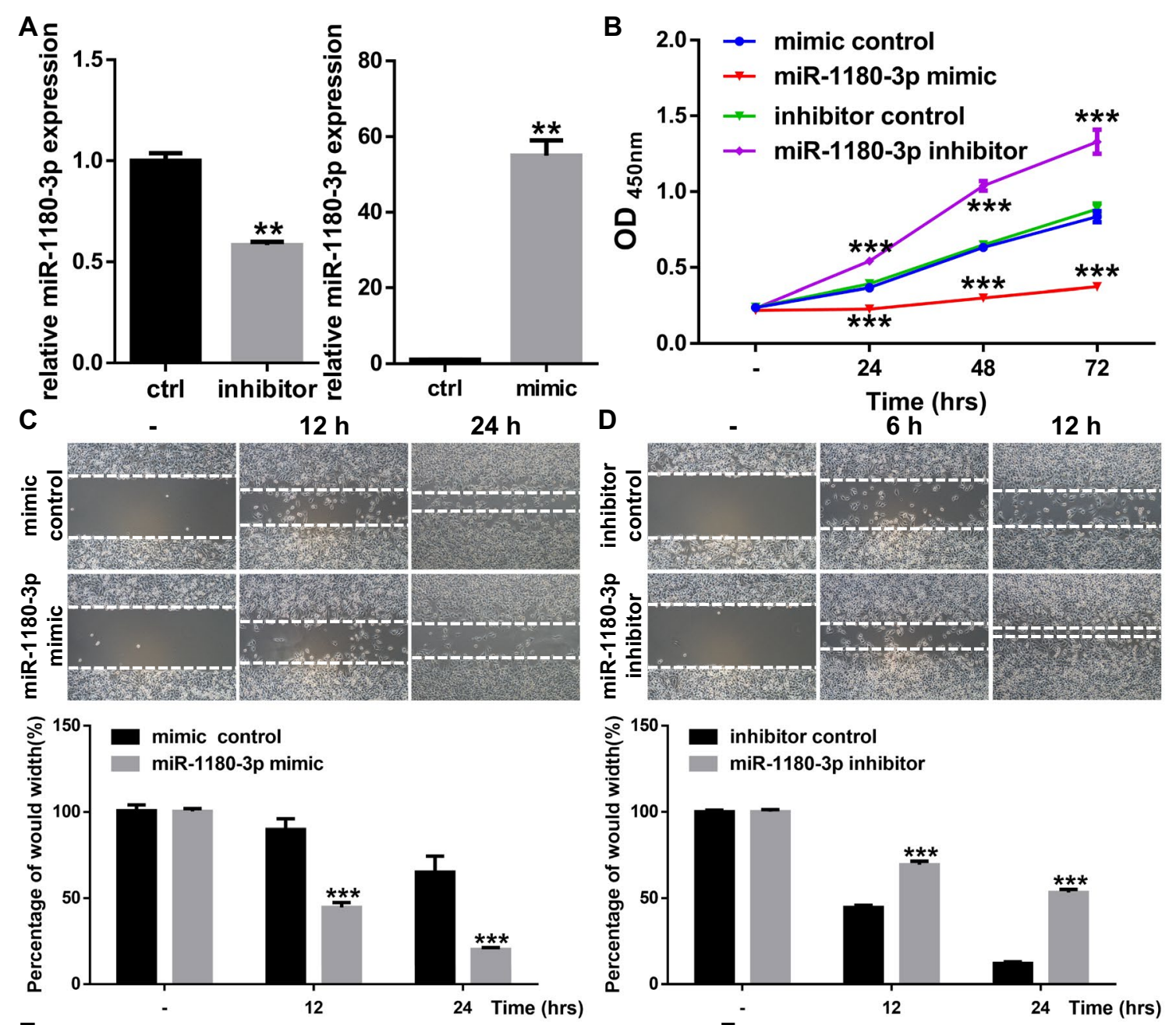

E
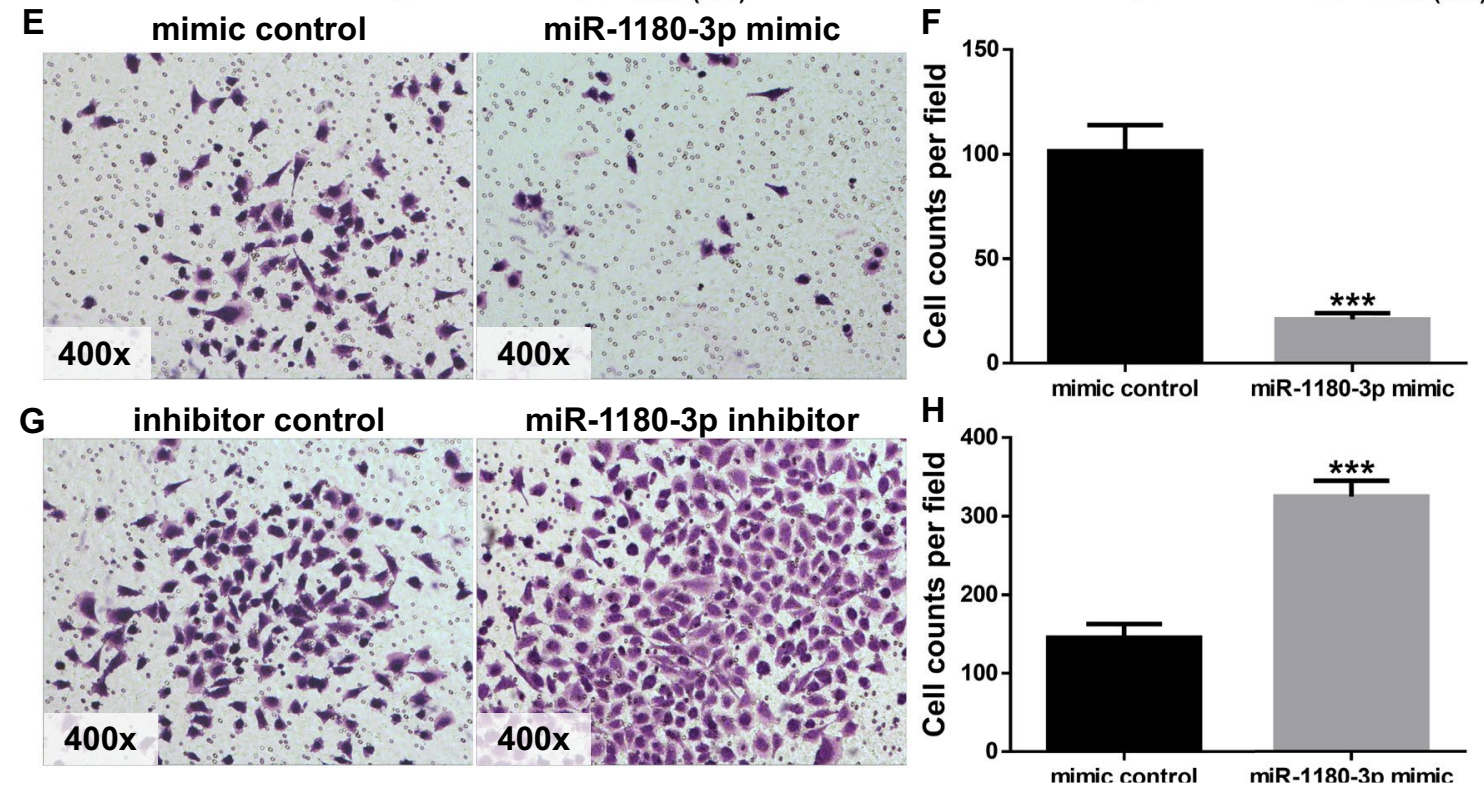

Fig. 4 (See legend on previous page.) 
in melanoma remains unclear. Therefore, the expression level of miR-1180-3p in melanoma cells was assessed by q-PCR. As expected, melanoma cells exhibited lower levels of miR-1180-3p than melanocytes (Fig. 3D).

\section{Decreased miR-1180-3p expression promotes the malignant phenotype of melanoma cells}

To better understand the biological role of miR-1180-3p, we generated miR-1180-3p-overexpressing or miR1180-3p-inhibited melanoma cells using an miRNA mimic or inhibitor (Fig. 4A). Interestingly, the growth of melanoma cells was remarkably negatively associated with the level of miR-1180-3p (Fig. 4B), which indicates that miR-1180-3p benefits the growth of melanoma cells. Consistent with this finding, the migration ability of melanoma cells was attenuated by the miR-1180-3p mimics (Fig. 4C). Accordingly, the miR-1180-3p inhibitors enhanced the migration ability of the cells (Fig. 4D). The number of invaded cells was negatively related to the miR-1180-3p expression in melanoma (Fig. 4E-H). These findings support that decreased miR-1180-3p expression promotes the malignant phenotype of melanoma cells.

\section{MAN2B1 and ST3GAL4 are potential target genes of miR-1180-3p and are negatively regulated by miR-1180-3p}

It is well established that miRNAs exert their function mainly by base pairing with target mRNAs to negatively regulate their expression [7]. For this reason, we used miRWalk [18], miRNA-Pathway [20] and ComiR [19] to predict the target genes of miR-1180-3p and eventually found 257 intersecting genes (Fig. 5A). Importantly, miR pathway analysis implied that miR-1180-3p is associated with the $\alpha$-MAN pathway (steps in the glycosylation of mammalian $\mathrm{N}$-linked oligosaccharides) in cutaneous melanoma. Therefore, we focused on 11 genes related to the $\alpha$-MAN pathway. We further examined the expression profiles of the selected genes by performing gene expression profiling interactive analysis (GEPIA) [21]. Among the 11 genes, MAN2B1, MAN2C1 and ST3GAL4 were significantly altered in melanoma patients compared to the normal controls (Fig. 5B-5D), while the other 8 genes were not significantly different between the patients and healthy controls (Fig. 5E-L). MAN2B1 and ST3GAL4 exhibited higher expression in melanoma patients (Fig. 5B, D), and MAN2C1 was downregulated in tumor tissues (Fig. 5C).

MAN2B1, a member of the alpha-mannosidase family, was reported to be closely related to alpha-mannosidosis [24]. The subcellular localization of mutant MAN2B1 is well known to be associated with inherited metabolic diseases [25], while the role of MAN2B1 in cancers is unknown. In addition to skin cutaneous melanoma (SKCM), the expression of MAN2B1 is generally high in other cancers, including cervical squamous cell carcinoma (CESC), esophageal carcinoma (ESCA), glioblastomas (GBM), kidney renal clear cell carcinoma (KIRC), brain lower-grade glioma (LGG), liver hepatocellular carcinoma (LIHC), and pancreatic adenocarcinoma (PAAD), as shown in Additional file 1: Figure S1A. Notably, MAN2B1 was expressed at the highest level in subjects with melanoma (Additional file 1: Figure S1B). Another alpha-mannosidase, MAN2C1, is downregulated in most tumors except thymoma (THYM), as shown in Additional file 2: Figure $\mathrm{S} 2 \mathrm{~A}$. Interestingly, MAN1C1 is known as a tumor suppressor in KIRC [26] and exerts protumor effects on several cancers. In ESCA, inhibition of MAN2C1 suppressed the proliferation of ESCA cells through mitotic arrest and apoptosis [27]. Moreover, MAN2C1 negatively regulates PTEN in prostate cancer, thereby promoting tumor development [28]. Among different cancers, MAN2C1 is moderately expressed in melanoma (Additional file 2: Figure S2B). Alteration of ST3GAL4 expression, a sialyltransferase that transfers sialic acid, has been implicated in carcinogenesis. Interestingly, its expression was dysregulated among different cancers, as shown in Additional file 3: Figure S3A. Interestingly, ST3GAL4 was specifically highly expressed in melanoma (Additional file 3: Figure S3B).

To determine the regulatory impacts of miR-1180-3p on MAN2B1, MAN2C1 and ST3GAL4, we detected the levels of MAN2B1, MAN2C1 and ST3GAL4 in SK-Mel-28 cells with miR-1180-3p overexpression or knockdown. Both MAN2B1 and ST3GAL4 were negatively regulated by miR1180-3p; however, the expression of MAN2C1 was only slightly increased after the overexpression of miR-1180-3p and was not significantly altered by the miR-1180-3p inhibitor (Fig. 5M). These results suggest that MAN2B1 and

\footnotetext{
(See figure on next page.)

Fig. 5 MAN2B1 and ST3GAL4 might be target genes of miR-1180-3p and negatively regulated by miR-1180-3p. A Venn diagram of predicted target genes. Target genes predicted by miRWalk, ComiR and miRNA-Pathway were analyzed as described in Methods. B-L Validation of MAN2B1, MAN2C1, ST3GAL4 B3GALT2, FUT5, FUT6, FUT7, FUT9, MAN1C1, MAN2A2 and ST8SIA4 from the expression analysis in GEPIA. The box plots are based on 461 melanoma samples (red) and 558 normal samples (gray), revealing the overexpression of MAN2B1 (B) and ST3GAL4 (D) and the decreased expression of MAN2C1 (C) in melanoma. M miR-1 180-3p negatively regulated the expression of MAN2B1 and ST3GAL4. RNA was extracted from melanoma cells transfected with mimic and inhibitor of miR-1180-3p. RT-Q-PCR was then performed with primers of MAN2B1 (left panel), MAN2C1 (middle panel) and ST3GAL4 (right panel) as described in the Methods. The data from multiple experiments $(n=3)$ are expressed as the mean \pm SD. Significant differences were evaluated using one-way ANOVA, and an asterisk $\left(^{*}\right)$ indicates a significant difference $\left({ }^{*} p<0.05,{ }^{* *} p<0.01,{ }^{* * *} p<0.001\right)$
} 


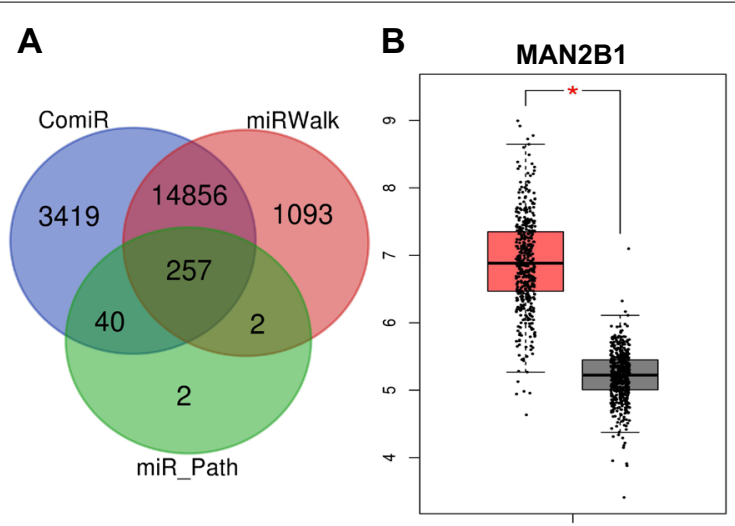

E
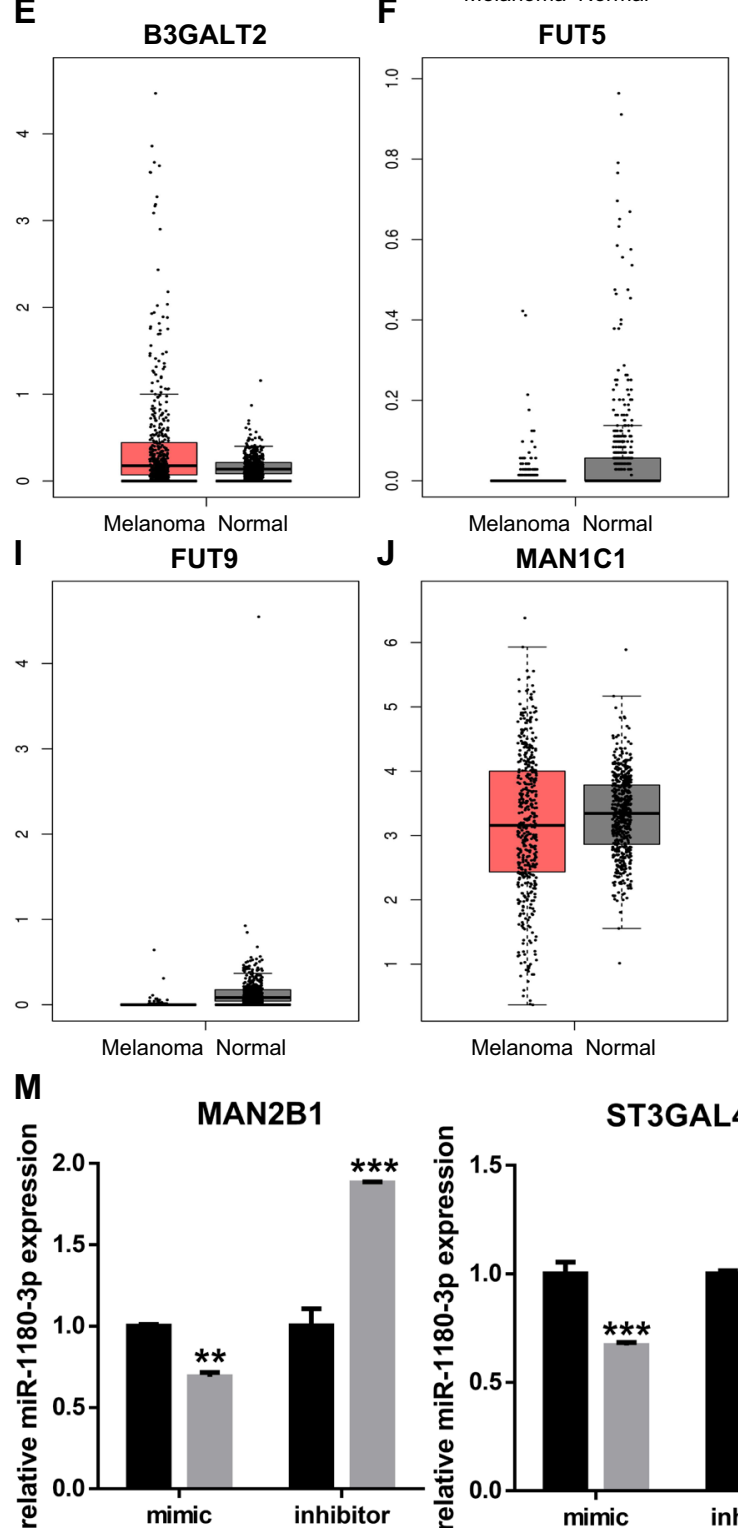
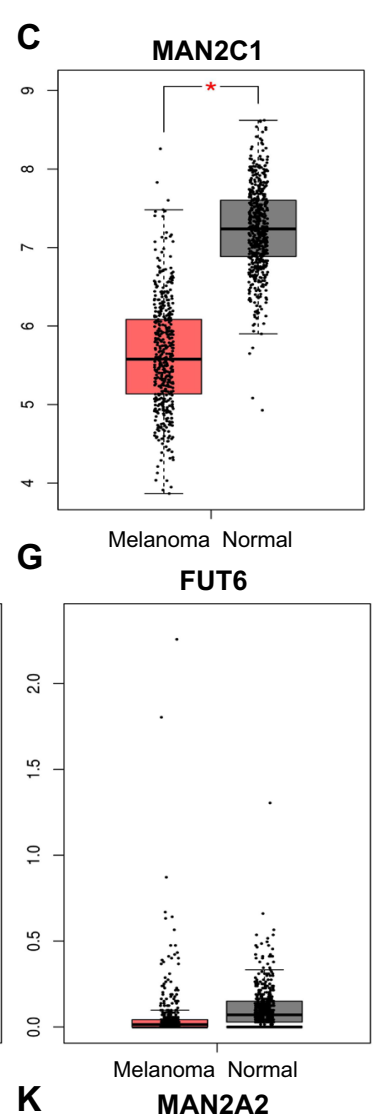
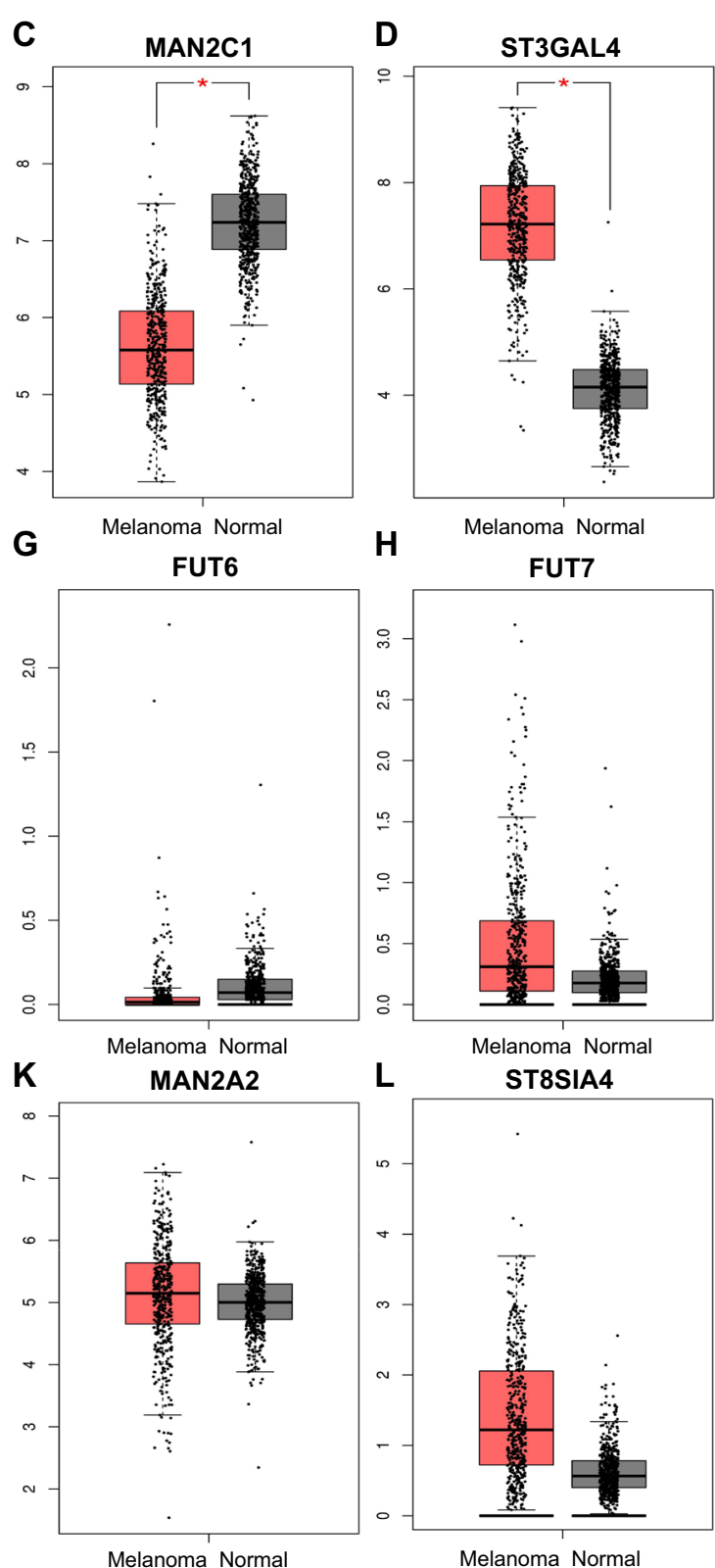

H Melanoma Normal

L ST8SIA4
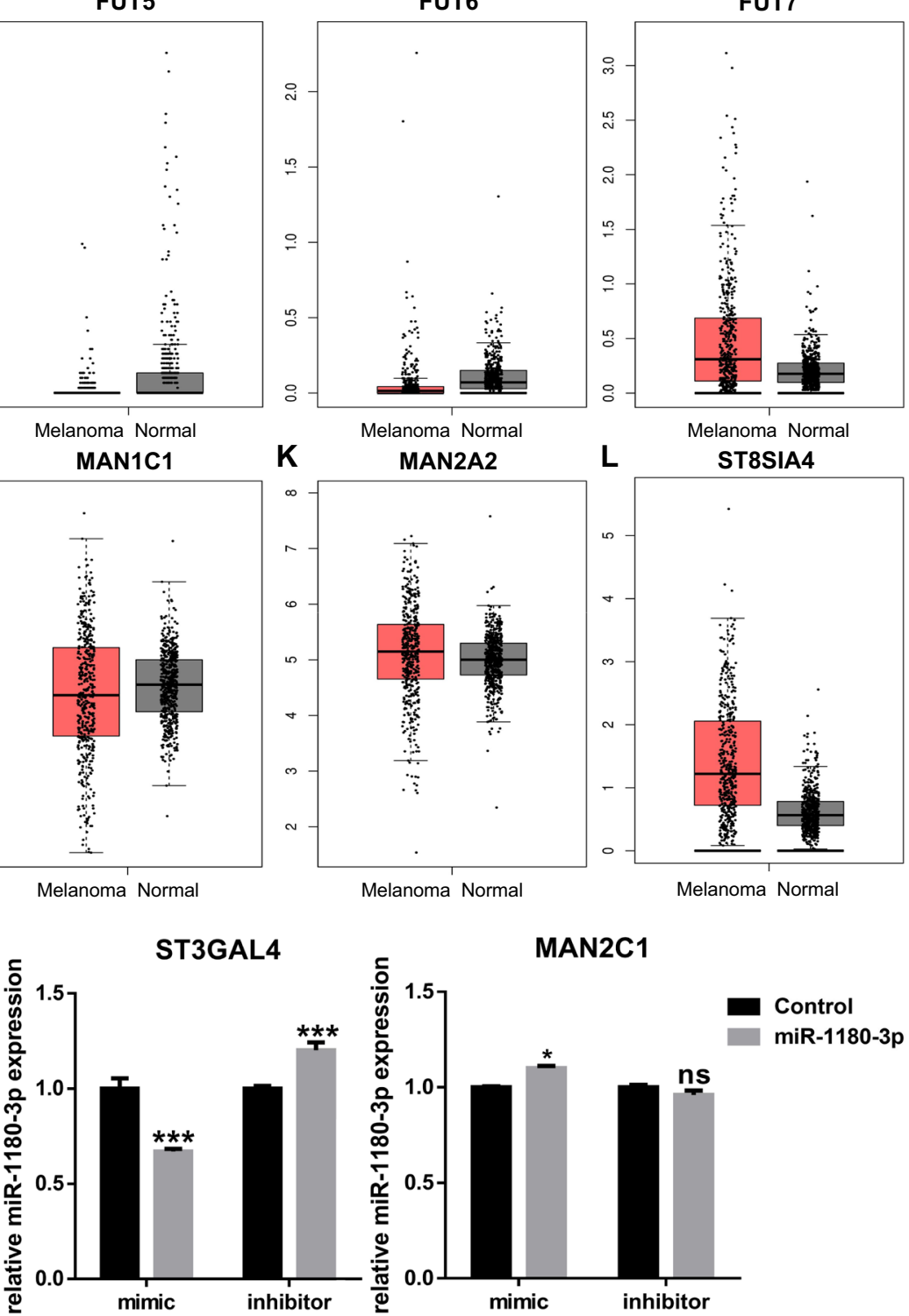

Fig. 5 (See legend on previous page.) 
ST3GAL4 might be the target genes and negatively regulated by miR-1180-3p.

\section{MiR-1180-3p binds to ST3GAL4 and regulates the malignant phenotype of melanoma cells}

We then predicted the miR-1180-3p binding sites on MAN2B1 and ST3GAL4 using RNAhybrid 2.2 and miRWalk. The results suggested that miR-1180-3p binds to the $3^{\prime} \mathrm{UTR}^{\prime}$ of ST3GAL4 and to the $5^{\prime} \mathrm{UTR}^{\prime}$ of MAN2B1. Considering that miRNAs are known to typically post-transcriptionally regulate target genes through the $3^{\prime}$-UTR [29], we constructed ST3GAL4 3'UTR-WT and ST3GAL4 3'UTR-Del luciferase reporter plasmids (Fig. 6A). The luciferase reporter assay results suggested that overexpression of miR1180-3p inhibited the expression of the ST3GAL4 3'UTR-WT promoter but not the ST3GAL4 3'UTRDel promoter (Fig. 6B), indicating that miR-1180-3p targets ST3GAL4.

To date, few studies have investigated the role of ST3GAL4 in the development of melanoma. Thus, we generated stable ST3GAL4-knockdown melanoma cells using short hairpin RNA (shRNA), as shown in Fig. 6C. The cell proliferation assay displayed inhibited growth after ST3GAL4 knockdown (Fig. 6D). Consistently, the number of migrated cells was remarkably reduced in ST3GAL4-deficient melanoma cells (Additional file 5: Figure S4). In addition, the invasion ability was attenuated when the expression of ST3GAL4 was inhibited in melanoma cells (Fig. 6E, F) (Additional file 5: Figure S4).

As miR-1180-3p might regulate the biological behavior of melanoma cells by targeting ST3GAL4, we treated ST3GAL4-deficient melanoma cells with
miR-1180-3p mimics and inhibitors. As expected, the Transwell assay showed no significant difference in the numbers of invaded cells treated with the miR-1180-3p mimics or inhibitors after knocking down ST3GAL4 (Fig. 6G, H) (Additional file 6: Figure S5), supporting our hypothesis that miR-1180-3p regulates the malignant phenotype of melanoma by targeting ST3GAL4.

Taken together, our study results demonstrated that miR-1180-3p expression was decreased in exosomes derived from melanoma patients compared with those from healthy donors and was thus identified as a prospective melanoma biomarker. In addition, decreased miR-1180-3p expression promoted the development of melanoma by targeting ST3GAL4.

\section{Discussion}

In this study, the exosomal miRNA expression profile from the plasma of melanoma patients was investigated. MiR-1180-3p expression was decreased in exosomes derived from melanoma patient plasma and exhibited diagnostic potential. In addition, decreased miR-1180-3p expression promoted the proliferation of melanoma cells by elevating ST3GAL4. To the best of our knowledge, this is the first plasma exosomal miRNA study on Chinese melanoma patients. These findings suggest a prospective strategy for the early diagnosis of melanoma and provide additional insight into melanoma development.

Exosomal miRNAs from plasma have been studied in various cancers with the aim of early diagnosis and improving knowledge of cancer biology. In breast cancer, certain exosomal microRNAs are upregulated in the plasma of patients, including miR-21 and miR-1246 [30]. For pancreatic cancer, exosomal miR-196a and miR-1246 are promising biomarkers [31]. In some cases,

\footnotetext{
(See figure on next page.)

Fig. 6 MiR-1180-3p binds to ST3GAL4 and regulate the malignant phenotype of melanoma cells. A Schematic diagram of predicted binding sites of ST3GAL4 with miR-1180-3p. The binding sites was predicted by RNAhybrid 2.2 and miRWalk as described in Methods. The predicted binding sites were deleted in the sequence (ST3GAL4-3'UTR-Del) based on ST3GAL4-3'UTR-WT luciferase reporter plasmids. B The luciferase activity of ST3GAL4 promoter was inhibited by miR-1180-3p. The luciferase reporter assay was performed in 293T cells transfected with ST3GAL4 3'UTR-WT or ST3GAL4 3'UTR-Del, and treated with miR-1 180-3p mimic or control as described in Methods. Data from multiple experiments are expressed as the mean $\pm S D(n=4)$. Significant differences were evaluated using two-way ANOVA, ${ }^{*} p<0.05,{ }^{* *} p<0.01,{ }^{* * *} p<0.001$. C Knockdown of ST3GAL4 in melanoma cells. Stable knockdown of ST3GAL4 in SK-MEL-28 cells was generated by lentiviral infection and subjected to RT-Q-PCR analysis as described in the Methods. Data from multiple experiments are expressed as the mean $\pm S D(n=3)$. Significant differences were evaluated using Student's t-test, ${ }^{*} p<0.05,{ }^{* *} p<0.01,{ }^{* * *} p<0.001$. D Knockdown of ST3GAL4 suppress the proliferation of SK-Mel-28. ST3GAL4-deficient SK-Mel-28 cells were seeded into 96-well plates, and cell viability was examined by CCK-8 kit as described in Methods. Data from multiple experiments are expressed as the means $\pm S D$. Significant differences were evaluated using Two-way ANOVA, ${ }^{*} p<0.05,{ }^{* *} p<0.01,{ }^{* * *} p<0.001$. E, F Inhibition of ST3GAL4 attenuates the invasion ability of SK-Mel-28 cells. Transwell assays were performed as described in Methods. Representative images were taken at indicated hours (E). The number of invasive cells per field was calculated, and the data was presented as the means $\pm S D(n=4)$ of each group $(\mathbf{F})$. The significant difference between cells was evaluated by Student's t-test. ${ }^{*} p<0.05,{ }^{* *} p<0.01,{ }^{* * *} p<0.001$. G, H The regulatory role of miR-1180-3p on melanoma cells were diminished after knocking down ST3GAL4. ST3GAL4-deficient melanoma cells were treated with miR-1 180-3p mimics or inhibitors. Transwell assays were performed in SK-Mel-5 (G) and SK-Mel-28 (H) as described in Methods. The number of invasive cells per field was calculated, and the data was presented as the means $\pm S D(n=4)$ of each group. The significant difference between cells was evaluated by Two-way ANOVA. ${ }^{*} p<0.05,{ }^{* *} p<0.01,{ }^{* * *} p<0.001$
} 


\section{A}

Position 49

ST3GAL4

3'UTR-WT 5' GACCCCCATGCGTGGCTGTGGGGG 3'

miR-1180-3p 3' GUGUGGGUGCGCUUCGGCUCUUU 5, $^{\mid}$

ST3GAL4

3'UT

C

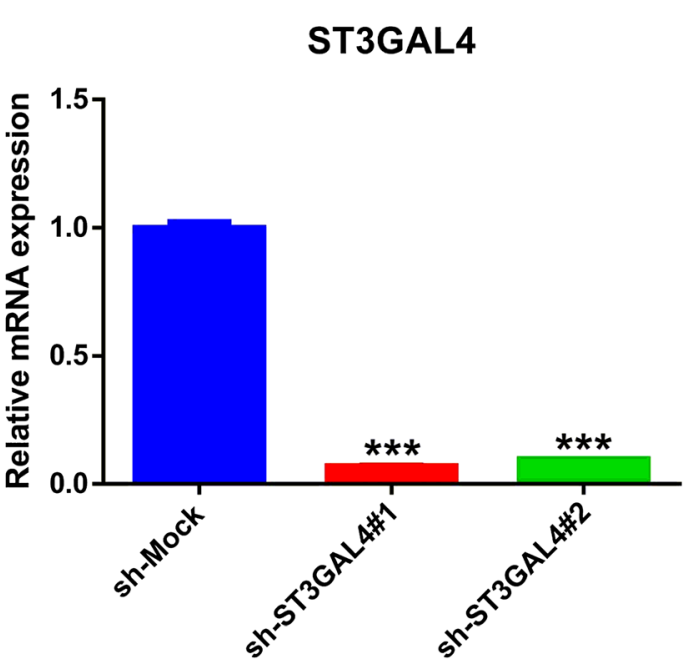

E sh-Mock

G 3'

D

B

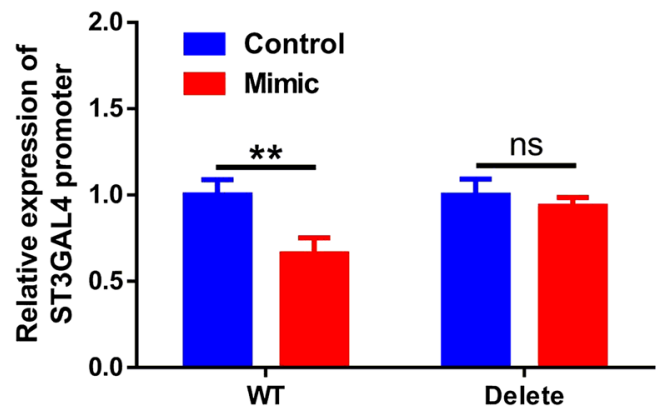

ST3GAL4

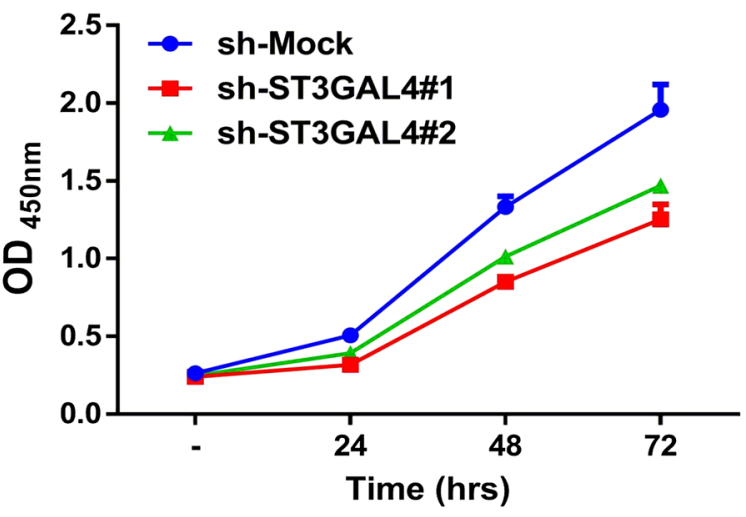

$\mathbf{F}$

ST3GAL4
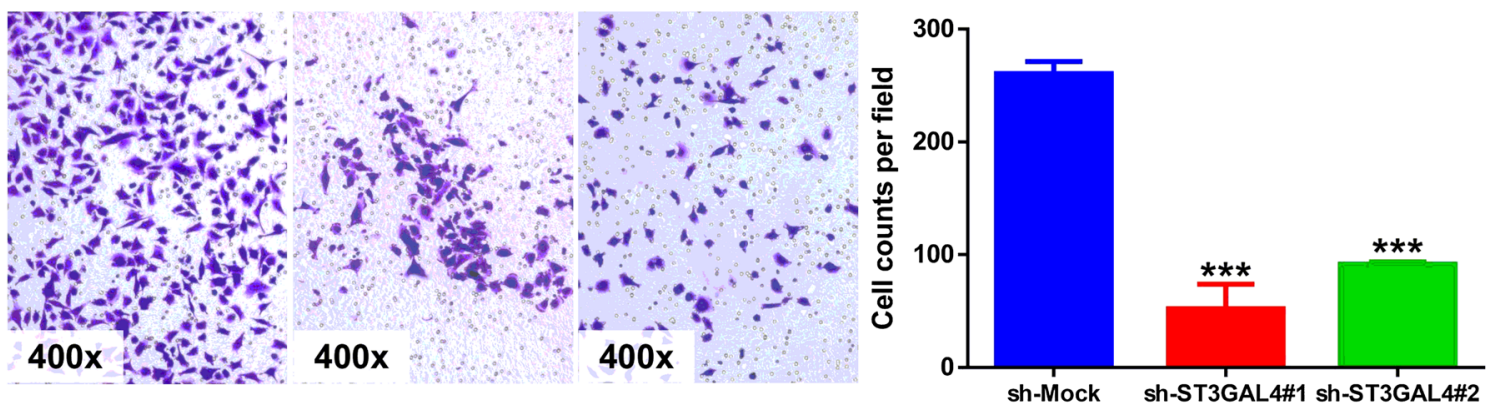

G

SK-Mel-5

H

SK-Mel-28
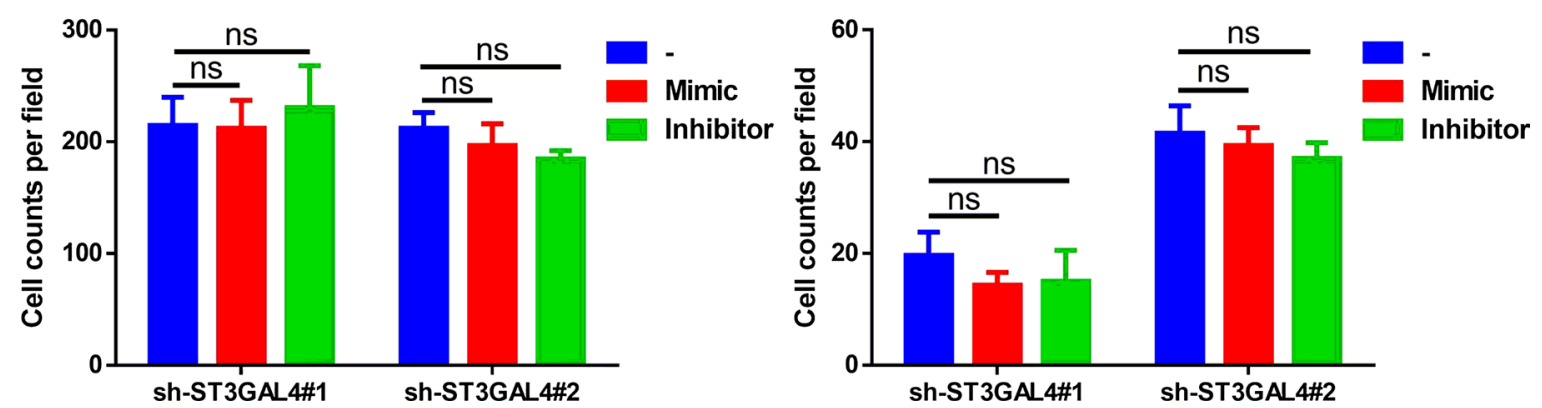

Fig. 6 (See legend on previous page.) 
plasma exosomal miRNAs serve as markers for differential diagnosis. For instance, miR-21 and miR-181a in plasma exosomes distinguish follicular from papillary thyroid cancer [32]. Different combinations of exosomal miRNAs differentiate adenocarcinoma from squamous cell carcinoma in the early diagnosis of NSCLC [33]. Exosomal miRNAs also have prognostic potential in cancer patients. Among different stages of colorectal cancer, increased miR-21 expression indicates metastasis and a poor prognosis [34]; higher levels of exosomal miR-1290 and miR-375 have been associated with poor overall survival in castration-resistant prostate cancer [35].

Attempts have been made to investigate the functions of exosomal miRNAs in melanoma. Exosomal miR-222 was shown to promote melanoma tumorigenesis [36]. Susan et al. identified several differentially regulated miRNAs derived from the plasma exosomes of patients with sporadic melanoma. However, the study did not show the efficacies of those miRNAs to diagnose melanoma. In addition, the differential miRNAs were analyzed by an miRNA array that included $\sim 700$ human miRNAs, and some important miRNAs might have been excluded [14]. An miRNA array was assembled that included exosomes derived from melanoma cell lines and indicated that specific miRNAs, including miR-31, miR-186, and miR$34 \mathrm{~b}$, are associated with melanoma invasion [37]. Here, our findings revealed the exosomal miRNA profile in the plasma of melanoma patients and revealed reduced miR1180-3p expression as a potential diagnostic marker for melanoma for the first time.

Studies have revealed the potential of miR-1180-3p in cancer diagnosis. The expression of miR-1180-3p in the blood was found to separate NSCLC patients from healthy individuals, with an AUC of 0.69 [38]. The circulatory plasma expression of miR-1180-3p, miR-425-5p, miR-122-5p, miR-24-3p and miR-4632-5p serves as a biomarker panel for the early detection of gastric cancer [39]. However, the function and mechanism of miR-1180-3p in melanoma have not yet been fully investigated. In the current study, we demonstrated that decreased miR-1180-3p expression supports the growth of melanoma cells and provide new insight into the role of miR-1180-3p in melanoma development.

The incidence rate of melanoma has increased by $110.3 \%$ in the last two decades in China [40]. For Asian melanoma patients, acral lentiginous melanoma, the most common subtype, accounts for $50 \%$ to $58 \%$ of cutaneous melanoma cases [41-43]. Usually, acral melanoma presents in the palms, soles, and nail apparatuses. Therefore, early tumors are difficult to notice and are typically diagnosed at a late stage, resulting in a poor prognosis [44]. Compared to the early stages of melanoma, advanced melanoma causes a significant economic burden $[45,46]$. These characteristics suggest that the early and timely diagnosis of melanoma is critical for improving prognosis and will help to reduce the global disease burden. Our findings herein demonstrate that plasma exosomal miR-1180-3p is a promising diagnostic biomarker for melanoma patients. Importantly, the miR-1130-3p/ST3GAL4 axis could be a therapeutic target for melanoma treatment.

\section{Abbreviations}

CESC: Cervical squamous cell carcinoma; ESCA: Esophageal carcinoma; GBM: Glioblastomas; KIRC: Kidney renal clear cell carcinoma; LGG: Brain lower-grade glioma; LIHC: Liver hepatocellular carcinoma; miRNA: MicroRNA; PAAD: Pancreatic adenocarcinoma.

\section{Supplementary Information}

The online version contains supplementary material available at https://doi. org/10.1186/s12935-021-02164-8.

Additional file 1: Figure S1. MAN2B1 is generally highly expressed in cancers. (A) MAN2B1 is generally highly expressed in cancers. The expression profile of MAN2B1 in different cancers was analyzed by GEPIA. MAN2B1 is overexpressed in CESC, DLBC, ESCA, GBM, KIRC, KIRP, LAML, LGG, LIHC, PAAD, SKCM, TGCT, UCEC and UC. (B) The expression level of MAN2B1 in melanoma is higher than that in other cancers. Comparison of MAN2B1 expression in different cancers was analyzed in ProteinAtlas as described in Methods.

Additional file 2: Figure S2. MAN2C1 is generally down-regulated in cancers. (A) MAN2C1 is generally down-regulated in cancers. The expression profile of MAN2C1 in different cancers was analyzed by GEPIA. MAN2B1 is overexpressed in ACC, BRCA, CESC, COAD, ESCA, GBM, LUAD, LUSC, OV, PRAD, READ, SKCM, STAD, TGCT, THCA, UCEC and UCS, but highly expressed in THYM. (B) The expression level of MAN2B1 in melanoma is moderate among different cancers. Comparison of MAN2C1 expression in different cancers was analysed in ProteinAtlas as described in Methods.

Additional file 3: Figure S3. ST3GAL4 is specifically highly expressed in melanoma. (A) ST3GAL4 is specifically highly expressed in melanoma. The expression profile of ST3GAL4 in different cancers was analyzed by GEPIA. ST3GAL4 is overexpressed in SKCM, LAML and PAAD, while down-regulated in DLBC, ESCA, KIRP, OV, TGCT and THYM. (B) The expression level of ST3GAL4 in melanoma is significantly higher than that in other cancers. Comparison of ST3GAL4 expression in different cancers was analyzed in ProteinAtlas as described in Methods.

Additional file 4: Table S1. The demographic and clinical information of participants.

Additional file 5: Figure S4. ST3GAL4 regulates the migration ability of melanoma cells. (A) Knockdown of ST3GAL4 inhibit the migration of SKMEL-28 cells. Migration assays were performed as described inMethods. Representative images were taken at indicated hours. (B) Bar chart of migration assay. The number of invasive cells per field was calculated, and the data was presented as the means $\pm S D(n=4)$ of eachgroup. The significant difference between cells was evaluated by Student's t-test. ${ }^{*} p<$ $0.05,{ }^{* *} \mathrm{p}<0.01,{ }^{* * *} \mathrm{p}<0.001$

Additional file 5: Figure S4. ST3GAL4 regulates the migration ability of melanoma cells. (A) Knockdown of ST3GAL4 inhibit the migration of SKMEL-28 cells. Migration assays were performed as described in Methods. Representative images were taken at indicated hours. (B) Bar chart of migration assay. The number of invasive cells per field was calculated, and the data was presented as the means $\pm S D(n=4)$ of each group. The significant difference between cells was evaluated by Student's t-test. ${ }^{*} p<0.05,{ }^{* *} p<0.01,{ }^{* * *} p<0.001$ 
Additional file 6: Figure S5. Knockdown of ST3GAL4 impairs the regulatory role of miR-1 180-3p on invasion ability of melanoma cells. (A-B) ST3GAL4-deficient melanoma cells were treated with miR-1180-3pmimics or inhibitors. Representative images of SK-Mel-5 (A) and SK-Mel-28 (B) were taken at indicated hours as described in Methods.

Additional file 6: Figure S5. Knockdown of ST3GAL4 impairs the regulatory role of miR-1 180-3p on invasion ability of melanoma cells. (A-B) ST3GAL4-deficient melanoma cells were treated with miR-1180-3p mimics or inhibitors. Representative images of SK-Mel-5 (A) and SK-Mel-28 (B) were taken at indicated hours as described in Methods.

\section{Acknowledgements}

We would like to thank all the melanoma patients who participated in our study for their support and assistance in the work.

\section{Authors' contributions}

YG analyzed and interpreted the patient data and was a major contributor in writing the manuscript. XZ performed in vitro studies. LW and $M L$ analyzed the sequencing data. MS was responsible for the statistical analysis. SZ, ZZ, SZ, $\mathrm{KL}, \mathrm{ZF}, \mathrm{BY}$ collected patient samples. JS provided the patient resources, CP and $X C$ conceptualized and supervised the study. All authors read and approved the final manuscript

\section{Funding}

This work was supported by the National Natural Science Foundation of China (81572679, 81773341, 81830096, 818142020, 81620108024, 82002911), Overseas Expertise Introduction Project for Discipline Innovation (111 Project, No. B20017), China Postdoctoral Science Foundation (2020T130729) and China Scholarship Council (201906370201).

\section{Availability of data and materials}

The datasets generated and/or analysed during the current study are available from the corresponding author on request.

\section{Declarations}

\section{Ethics approval and consent to participate}

This study was approved by the Institutional Research Ethics Boards of Xiangya Hospital, Central South University. All the donors have signed the consent forms.

\section{Consent for publication}

Not applicable.

\section{Competing interests}

The authors declare that they have no competing interests.

\section{Author details}

'Department of Dermatology, Xiangya Hospital, Central South University, Xiangya Road \#87, Changsha 410008, China. ${ }^{2}$ Hunan Key Laboratory of Skin Cancer and Psoriasis, Hunan Engineering Research Center of Skin Health and Disease, Xiangya Hospital, Central South University, Changsha 410008, China. ${ }^{3}$ National Clinical Research Center for Geriatric Disorders, Xiangya Hospital, Central South University, Changsha 410008, China. ${ }^{4}$ Hunan Provincial Key Lab on Bioinformatics, School of Computer Science and Engineering, Central South University, Changsha 410083, China.

\section{Received: 19 March 2021 Accepted: 19 August 2021}

Published online: 20 September 2021

\section{References}

1. Liu C, Yang Y, Wu Y. Recent advances in exosomal protein detection via liquid biopsy biosensors for cancer screening, diagnosis, and prognosis. AAPS J. 2018;20(2):41.
2. Palmirotta R, Lovero D, Cafforio P, Felici C, Mannavola F, Pelle E, Quaresmini D, Tucci M, Silvestris F. Liquid biopsy of cancer: a multimodal diagnostic tool in clinical oncology. Ther Adv Med Oncol. 2018;10:1758835918794630.

3. Li M, Zeringer E, Barta T, Schageman J, Cheng A, Vlassov AV. Analysis of the RNA content of the exosomes derived from blood serum and urine and its potential as biomarkers. Philos Trans R Soc Lond B Biol Sci. 2014;369(1652):20130502.

4. Kalluri R, LeBleu VS. The biology, function, and biomedical applications of exosomes. Science. 2020;367(6478):eaau6977.

5. Kalluri R. The biology and function of exosomes in cancer. J Clin Invest. 2016;126(4):1208-15.

6. Pathan M, Fonseka P, Chitti SV, Kang T, Sanwlani R, Van Deun J, Hendrix A, Mathivanan S. Vesiclepedia 2019: a compendium of RNA, proteins, lipids and metabolites in extracellular vesicles. Nucleic Acids Res. 2019;47(D1):D516-9.

7. Macfarlane LA, Murphy PR. MicroRNA: biogenesis, function and role in cancer. Curr Genomics. 2010;11(7):537-61.

8. Wang M, Yu F, Ding H, Wang Y, Li P, Wang K. Emerging function and clinical values of exosomal microRNAs in cancer. Mol Ther Nucleic Acids. 2019;16:791-804

9. Rahbarghazi R, Jabbari N, Sani NA, Asghari R, Salimi L, Kalashani SA, Feghhi M, Etemadi T, Akbariazar E, Mahmoudi M, et al. Tumor-derived extracellular vesicles: reliable tools for Cancer diagnosis and clinical applications. Cell Commun Signal. 2019;17(1):73.

10. Ahmadi M, Jafari R, Mahmoodi M, Rezaie J. The tumorigenic and therapeutic functions of exosomes in colorectal cancer: opportunity and challenges. Cell Biochem Funct. 2021;39(4):468-77.

11. Miller AJ, Mihm MC Jr. Melanoma. N Engl J Med. 2006;355(1):51-65.

12. Surveillance $E$, and End Results (SEER) Program (www.seer.cancer.gov). In.; Nov 2019 Sub (1975-2017)

13. Houghton AN, Polsky D. Focus on melanoma. Cancer Cell. 2002;2(4):275-8.

14. Pfeffer SR, Grossmann KF, Cassidy PB, Yang CH, Fan M, Kopelovich L, Leachman SA, Pfeffer LM. Detection of exosomal miRNAs in the plasma of melanoma patients. J Clin Med. 2015;4(12):2012-27.

15. Lee HY, Chay WY, Tang MB, Chio MT, Tan SH. Melanoma: differences between Asian and Caucasian patients. Ann Acad Med Singapore. 2012;41(1):17-20.

16. Chong T, YX, Wei Yan: AASRA: An anchor alignment-based small RNA annotation pipeline. BioRxiv 2017, 132928.

17. Wang L, Feng Z, Wang X, Wang X, Zhang X. DEGseq: an R package for identifying differentially expressed genes from RNA-seq data. Bioinformatics. 2010;26(1):136-8.

18. Dweep H, Gretz N. miRWalk2.0: a comprehensive atlas of microRNAtarget interactions. Nat Methods. 2015;12(8):697.

19. Coronnello C, Benos PV. ComiR: combinatorial microRNA target prediction tool. Nucleic Acids Res. 2013:41:W159-164.

20. Ma Z, Liu T, Huang W, Liu H, Zhang HM, Li Q, Chen Z, Guo AY. MicroRNA regulatory pathway analysis identifies miR-142-5p as a negative regulator of TGF-beta pathway via targeting SMAD3. Oncotarget. 2016;7(44):71504-13.

21. Tang Z, Li C, Kang B, Gao G, Li C, Zhang Z. GEPIA: a web server for cancer and normal gene expression profiling and interactive analyses. Nucleic Acids Res. 2017:45(W1):W98-102.

22. Zhou Z, Zhou X, Jiang Y, Qiu M, Liang X, Lin Q, Guo Q, Nong C, Huo R, Chen $Q$, et al. Clinical significance of miR-1180-3p in hepatocellular carcinoma: a study based on bioinformatics analysis and RT-qPCR validation. Sci Rep. 2020;10(1):11573.

23. Chen M, Huang X, Li L, Huang M, Cai R, Liao X. A regulatory axis of circ_0008193/miR-1180-3p/TRIM62 suppresses proliferation, migration, invasion, and Warburg effect in lung adenocarcinoma cells under hypoxia. Med Sci Monit. 2020;26:e922900.

24. Riise Stensland HM, Klenow HB, Nguyen LV, Hansen GM, Malm D, Nilssen O. Identification of 83 novel alpha-mannosidosis-associated sequence variants: functional analysis of MAN2B1 Missense mutations. Hum Mutat. 2016;37(8):827.

25. Borgwardt L, Stensland HM, Olsen KJ, Wibrand F, Klenow HB, Beck M, Amraoui Y, Arash L, Fogh J, Nilssen O, et al. Alpha-mannosidosis: correlation between phenotype, genotype and mutant MAN2B1 subcellular localisation. Orphanet J Rare Dis. 2015;10:70. 
26. Li H, Wang G, Yu Y, Jian W, Zhang D, Wang Y, Wang T, Meng Y, Yuan C, Zhang C. alpha-1,2-Mannosidase MAN1C1 inhibits proliferation and invasion of clear cell renal cell carcinoma. J Cancer. 2018;9(24):4618-26.

27. Tian Y, Ju JY, Zhou YQ, Liu Y, Zhu LP. Inhibition of alpha-mannosidase Man2c1 gene expression suppresses growth of esophageal carcinoma cells through mitotic arrest and apoptosis. Cancer Sci. 2008;99(12):2428-34.

28. He L, Fan C, Kapoor A, Ingram AJ, Rybak AP, Austin RC, Dickhout J, Cutz JC, Scholey J, Tang D. alpha-Mannosidase 2C1 attenuates PTEN function in prostate cancer cells. Nat Commun. 2011;2:307.

29. Lee I, Ajay SS, Yook JI, Kim HS, Hong SH, Kim NH, Dhanasekaran SM, Chinnaiyan AM, Athey BD. New class of microRNA targets containing simultaneous 5'-UTR and 3'-UTR interaction sites. Genome Res. 2009;19(7):1175-83.

30. Hannafon BN, Trigoso YD, Calloway CL, Zhao YD, Lum DH, Welm AL, Zhao ZJ, Blick KE, Dooley WC, Ding WQ. Plasma exosome microRNAs are indicative of breast cancer. Breast Cancer Res. 2016;18(1):90.

31. XU YF, Hannafon BN, Zhao YD, Postier RG, Ding WQ. Plasma exosome miR$196 a$ and miR-1246 are potential indicators of localized pancreatic cancer. Oncotarget. 2017;8(44):77028-40.

32. Samsonov R, Burdakov V, Shtam T, Radzhabovsmall CZ, Vasilyev D, Tsyrlina E, Titov S, Ivanov M, Berstein L, Filatov M, et al. Plasma exosomal miR-21 and miR-181a differentiates follicular from papillary thyroid cancer. Tumour Biol. 2016;37(9):12011-21.

33. Jin X, Chen Y, Chen H, Fei S, Chen D, Cai X, Liu L, Lin B, Su H, Zhao L, et al. Evaluation of tumor-derived exosomal miRNA as potential diagnostic biomarkers for early-stage non-small cell lung cancer using next-generation sequencing. Clin Cancer Res. 2017;23(17):5311-9.

34. Tsukamoto M, linuma H, Yagi T, Matsuda K, Hashiguchi Y. Circulating exosomal microRNA-21 as a biomarker in each tumor stage of colorectal cancer. Oncology. 2017;92(6):360-70.

35. Huang X, Yuan T, Liang M, Du M, Xia S, Dittmar R, Wang D, See W, Costello BA, Quevedo F, et al. Exosomal miR-1290 and miR-375 as prognostic markers in castration-resistant prostate cancer. Eur Urol. 2015;67(1):33-41.

36. Felicetti F, De Feo A, Coscia C, Puglisi R, Pedini F, Pasquini L, Bellenghi M, Errico MC, Pagani E, Care A. Exosome-mediated transfer of miR-222 is sufficient to increase tumor malignancy in melanoma. J Transl Med. 2016;14:56
37. Xiao D, Ohlendorf J, Chen Y, Taylor DD, Rai SN, Waigel S, Zacharias W, Hao $\mathrm{H}$, McMasters KM. Identifying mRNA, microRNA and protein profiles of melanoma exosomes. PLoS ONE. 2012;7(10):e46874.

38. Leidinger P, Brefort T, Backes C, Krapp M, Galata V, Beier M, Kohlhaas J, Huwer H, Meese E, Keller A. High-throughput qRT-PCR validation of blood microRNAs in non-small cell lung cancer. Oncotarget. 2016;7(4):4611-23.

39. Zhu XL, Ren LF, Wang HP, Bai ZT, Zhang L, Meng WB, Zhu KX, Ding $\mathrm{FH}$, Miao L, Yan J, et al. Plasma microRNAs as potential new biomarkers for early detection of early gastric cancer. World J Gastroenterol. 2019;25(13):1580-91.

40. Wu Y, Wang Y, Wang L, Yin P, Lin Y, Zhou M. Burden of melanoma in China, 1990-2017: findings from the 2017 global burden of disease study. Int J Cancer. 2019;147(3):692-701.

41. Chi Z, Li S, Sheng X, Si L, Cui C, Han M, Guo J. Clinical presentation, histology, and prognoses of malignant melanoma in ethnic Chinese: a study of 522 consecutive cases. BMC Cancer. 2011;11:85.

42. Ishihara K, Saida T, Otsuka F, Yamazaki N. Prognosis, Statistical Investigation Committee of the Japanese Skin Cancer S: statistical profiles of malignant melanoma and other skin cancers in Japan: 2007 update. Int J Clin Oncol. 2008;13(1):33-41.

43. Jung HJ, Kweon SS, Lee JB, Lee SC, Yun SJ. A clinicopathologic analysis of 177 acral melanomas in Koreans: relevance of spreading pattern and physical stress. JAMA Dermatol. 2013;149(11):1281-8.

44. Chang JW. Acral melanoma: a unique disease in Asia. JAMA Dermatol. 2013;149(11):1272-3.

45. Seidler AM, Pennie ML, Veledar E, Culler SD, Chen SC. Economic burden of melanoma in the elderly population: population-based analysis of the surveillance, epidemiology, and end results (SEER)-medicare data. Arch Dermatol. 2010;146(3):249-56.

46. Grange F, Mohr P, Harries M, Ehness R, Benjamin L, Siakpere O, Barth J, Stapelkamp C, Pfersch S, McLeod LD, et al. Economic burden of advanced melanoma in France, Germany and the UK: a retrospective observational study (Melanoma Burden-of-Illness Study). Melanoma Res. 2017;27(6):607-18.

\section{Publisher's Note}

Springer Nature remains neutral with regard to jurisdictional claims in published maps and institutional affiliations.
Ready to submit your research? Choose BMC and benefit from:

- fast, convenient online submission

- thorough peer review by experienced researchers in your field

- rapid publication on acceptance

- support for research data, including large and complex data types

- gold Open Access which fosters wider collaboration and increased citations

- maximum visibility for your research: over $100 \mathrm{M}$ website views per year

At BMC, research is always in progress.

Learn more biomedcentral.com/submissions 